\title{
Le passage de haies : de la réalité sportive en heptathlon à la réalité subjective d'Emma
}

\author{
M. Nicolas a,b,*, J. Jean b \\ a Faculté des sciences du sport de Bourgogne \\ b Association nationale des psychologues du sport (Anaps)
}

\section{Résumé}

Ce compte rendu de pratique souhaite souligner l'importance du suivi psychologique avec des adolescents sportifs. Le cas d'Emma illustre les transitions inhérentes de cette période d'age dans le contexte spécifique du sport de haut niveau. Pour Emma, le passage de haie devient la métaphore de son conflit psychique. La contre performance qui se manifeste dans le passage de haie signale son ambivalence face à son histoire personnelle au sein des réalités des cultures sportives. Cette mise en sens de la problématique sportive dans le cadre du suivi psychologique a pu l'aider à dépasser ses difficultés de passage et favoriser son engagement comme sujet de son propre désir sur la voie de sa réalisation dans l'acte sportif.

\section{Abstract}

The major dimension of this article is to point out the importance of psychological follow-up with sporting teenagers. Emma's example illustrates the inherent transitions from this period of age in the specific context of the high level sport. For Emma, the passage of hurdle becomes the metaphor of its psychical conflict. The counter performance which appears in the passage of hurdle announces its ambivalence with regard to its personal history within realities of sport cultures. This making sense of the sporting problems within the framework of the psychological follow-up can help to exceed this difficulty of passage and to support its engagement like subject of its own desire on the way of its realization in the sporting act

Mots clés : Préparation psychologique ; Suivi psychologique ; Transitions ; Adolescence ; Sport

Keywords: Psychological preparation; Psychological follow-up; Transitions; Adolescence; Sport

\section{Introduction}

Les pratiques professionnelles du psychologue se sont multipliées au sein de champs d'intervention diversifiés. Le sport de haut niveau est un de ces nouveaux champs d'investigation abordé par un domaine en psychologie maintenant reconnu : la psychologie du sport. Difficile au début, l'implication du psychologue dans le milieu sportif est sans cesse croissante à l'image des demandes des différents protagonistes du milieu sportif. Les modalités d'intervention du psychologue du sport sont en pleine définition et les dispositifs psychologiques restent à déterminer en fonction de chaque contexte d'intervention et de chaque problématique individuelle. Inscrit dans une démarche de recherche action, le but de cet article est de rendre compte d'une prise en charge psychologique dans le sport de haut niveau à partir d'une étude de cas dans le but d'engager une réflexion sur les pratiques qui 
restent à créer dans ce champ récent de la psychologie. Le cas d'Emma est emblématique d'une adolescente sportive de haut niveau2. Il illustre une problématique très largement abordée celui de l'adolescence et des transitions inhérentes à cette période de vie. Nous le commenterons dans le contexte particulier de l'adolescent sportif auquel se surajoutent des contraintes particulières liées au sport de compétition de haut niveau qui rendent ce passage encore plus spécifique. De plus, il faut noter que cette intervention de terrain s'inscrit dans le cadre d'une préparation psychologique.

La préparation psychologique est un des secteurs d'activité de la psychologie du sport appliquée3. Elle supporte différentes appellations qui sont fonction des références théoriques, des modèles utilisés ainsi que des données culturelles et des représentations des acteurs concernés. Ces modalités d'intervention recouvrent souvent diverses acceptions intéressantes à préciser car elles sous-tendent des pratiques d'intervention différentes (Nicolas, 2004). On oppose fréquemment les termes de préparation mentale à celui de préparation psychologique. La préparation mentale concerne principalement l'utilisation de techniques standardisées à l'approche de l'échéance de la compétition. Dans une perspective d'apprentissage, ces techniques ont pour but de favoriser l'acquisition d'habiletés psychologiques (Nideffer, 1981 ; Orlick, 1986 ; Unesthahl, 1983). Plus à distance de la compétition, la préparation psychologique cible la mobilisation de l'ensemble des ressources afin de favoriser une meilleure adaptation intrapsychique et interpsychique de l'individu à son environnement humain, matériel et physique. Ce terme implique un « ensemble coordonné de procédures directes ou indirectes, susceptibles d'améliorer le potentiel psychique de l'athlète » (Lévêque, 1993). Cet ensemble de procédures se traduit par de nombreuses pratiques développées dans le cadre de théories et méthodologies multiples comme les approches clinique, comportementale et cognitive, destinées à créer les conditions d'un investissement sportif efficace (Greenspan et Feltz, 1989 ; Vealey, 1994).

\section{Une psychologie appliquée au sport}

Le dispositif exposé inclut ces différentes modalités d'intervention : un suivi psychologique centré sur des entretiens individuels d'investigation et une préparation mentale avec l'application de techniques. Dans une perspective clinique, le suivi psychologique est proposé sous forme d'entretiens individuels afin que le sportif puisse investir par la parole et mettre en sens ses conflits psychiques pour les dépasser dans le cadre du dispositif de préparation psychologique. La deuxième modalité consiste en l'application d'une technique de préparation mentale, en l'occurrence la mise en place de séances d'imagerie mentale. La préparation mentale a pour but de développer les habiletés psychologiques susceptibles d'encourager également le dépassement des difficultés transitoires. La technique d'imagerie utilisée a été adaptée pour permettre de développer les habiletés techniques. Dans le cas d'Emma, il nous a paru judicieux de combiner ces approches avec d'une part, une prise de conscience et une élaboration de la dimension imaginaire et symbolique de la problématique psychologique, et d'autre part dans le cadre de la préparation mentale, l'apprentissage d'habiletés psychologiques pouvant également favoriser le dépassement des difficultés transitoires. Cet article se focalisera sur le suivi psychologique centré au niveau symbolique sur un travail d'élaboration psychique des changements auxquels est confrontée Emma.

\section{Emma : une adolescente sportive de haut niveau}

Le cas exposé ne relève pas d'une psychopathologie, mais de difficultés de passage communes à beaucoup de personnes de cette catégorie d'âge. Ces difficultés sont contextualisées dans le milieu sportif de haut niveau qui ajoute certaines contraintes 
spécifiques à ce milieu. Bien que singulière, cette vignette clinique peut être paradigmatique d'adolescent dans un environnement sportif de haut niveau. Elle illustre les difficultés de passage liées à la crise d'adolescence déplacée dans le contexte particulier de la compétition de haut niveau. Pour la troisième saison consécutive, le dispositif de préparation psychologique est présenté en début de saison à l'ensemble des membres du pôle 4. Suite à cette présentation, Emma rejoint d'elle-même le dispositif. Elle s'approprie d'emblée la démarche de manière active. D'origine étrangère, adoptée par des parents français lorsqu'elle était enfant, Emma a intégré le pôle France sur les conseils de l'entraîneur national contacté par ses parents. Elle réside au centre d'entrânement comme interne depuis son arrivée. Cette première année a été difficile, ses parents et ses amis lui manquent : «ici j'ai des collègues, c'est pas vraiment des amis ».bSon temps «libre » comme elle dit et ses moyens financiers sont limités. De plus en tant que mineure, le règlement intérieur du pôle ne lui permet pas de sortir comme elle le souhaiterait. Cependant, ses parents sont favorables à ce changement qui devrait selon eux favoriser ses progrès et son indépendance. Âgée de 17 ans, elle a atteint rapidement le niveau national avec un potentiel important qui peut lui permettre de disputer les championnats d'Europe dans sa catégorie malgré une pratique récente. Quant à ses projets scolaire et sportif, Emma suit des études techniques dans le secteur tertiaire dans l'intention de devenir comptable. Son projet sportif est de devenir athlète de haut niveau, ce projet est celui qui lui tient plus à cœur. Elle rejoint le pôle France décathlon avec l'ambition de faire partie de l'équipe nationale et de participer aux championnats de France. Selon son entourage sportif, elle représente le futur grand espoir français «si elle s'accroche ». Son entraîneur estime que son potentiel est considérable, mais qu'elle est « trop gentille, un peu tête en l'air » et qu'elle se démotive vite en cas de contre-performance. Dans ce discours qui sans stigmatiser l'athlète pourrait paraître prescriptif dans un libellé figé de ses compétences, l'entraîneur pense que si ses capacités physiques sont importantes, son «mental » peut l'empêcher d'atteindre les objectifs qui pourraient être les siens.

\subsection{La phase initiale : analyse de la demande et identification de la problématique sportive}

Elle manifeste d'emblée une certaine aisance. Lors du premier entretien, elle semble détendue et souriante. Sa demande initiale est vague. Elle attribue ses bons résultats à ses qualités physiques, mais elle considère avoir des lacunes au niveau technique : " J'ai de la chance, j'ai un bon physique mais j'ai pas de technique. Je suis venue au pôle pour améliorer ma technique ». Si elle pense bénéficier de conditions favorables pour progresser au sein du centre d'entraînement au niveau technique, son manque de résultat l'inquiète plus quand elle n'a pas ceux escomptés. «Je perds mes marques quand j'ai de mauvais résultats, je pense qu'à ça, alors que quand je bats mes records, je pense à rien. Sinon, je me prends la tête. J'ai peur de pas pouvoir avoir de meilleures performances et ça me stresse. »Cette identification à ces résultats génère un trouble. Questionnée sur ce qu'elle entend par perte de ses marques et comment elle se manifeste, dans quelles conditions elle la ressent le plus et dans quelles conditions elle la gère mieux, elle approfondit son investigation. De ces premiers entretiens d'explicitation, il résulte que l'épreuve lui posant principalement problème est le $100 \mathrm{~m}$ haie : «j'ai du mal aux haies ». L'énonciation de ses difficultés lui fait préciser : "C'est le passage de haie qui me stresse. J'arrive pas à relancer. » Après une reformulation, elle confirme : «En fait tout le passage de haie, dès l'attaque, le passage, la réception et la relance... Tout est lié. » Ces premiers entretiens nous semblent annonciateurs d'un lien entre la problématique sportive et la problématique psychologique. 


\subsection{Le passage de haies en heptathlon : un point de butée psychologique ?}

Pour les épreuves combinées, l'enchaînement des différentes épreuves est primordial. L'heptathlon est considéré comme un tout et non pas comme des épreuves séparées. L'important est de faire le lien. Fréquemment les spécialistes de l'heptathlon ont des épreuves fortes et des épreuves faibles. Parfois, ces dernières compromettent à tel point le décompte final de l'ensemble de la compétition qu'ils sont démotivés jusqu'à l'abandon. Les haies sont considérées comme une des épreuves les plus techniques et difficiles. De l'avis des techniciens, le passage de haies représente le moment critique du $100 \mathrm{~m}$ haie. Pour l'entraîneur, cet obstacle renforce les contraintes proposées à l'athlète. Cette succession d'obstacle doit être franchie sans à-coup, en fluidité dans le mouvement de la course.

Dans la réalité sportive, ce qui se passe en l'air, en suspension, se joue avant à l'impulsion. La reprise du passage est bonne si l'athlète a suffisamment de vitesse à l'impulsion. L'impulsion est la base de tout pour l'entraîneur, elle est à l'origine des difficultés de passage et de reprise du saut. Le travail technique sur le terrain consiste à changer l'intention de reprise derrière la haie, à changer la représentation du franchissement. La haie n'est plus le principal, comme le dit Emma : «Ce n'est plus l'obstacle que je dois franchir, mais je dois repartir derrière la haie, comme un nouveau start, un nouveau départ ». Ce qui implique d'augmenter la vitesse à l'impulsion pour en garder derrière la haie et donc ne pas piétiner avant l'obstacle, ni hésiter, ni douter, sinon la perte de vitesse compromet le passage. Le passage est ainsi dépendant de l'impulsion, tout se joue auparavant.

Cette réalité sportive fait écho au vécu subjectif de l'athlète. En effet, le présent s'origine dans le passé. Il faut se projeter dans le futur pour bien réussir le passage présent, et ainsi se propulser dans la relance. Cette conjugaison des temporalités sportives est à relier avec celles des processus psychologiques dans l'articulation du temps objectif sportif à celui plus subjectif de l'athlète. Dans la réalité psychique, Emma exprime ne pas retrouver ses repères. Dans cet enchaînement d'obstacle se produit la rupture dans la continuité, de la course qui s'accompagne d'un état de crise, d'un blocage qui la met face pas seulement à une haie mais la confronte à ses choix, ses points de butée, ses paradoxes et ses doutes. Nous émettons l'hypothèse que ce saut vers l'inconnu, vers le désir de performance, la convoque au sens de son existence et au déplacement de ses conflits psychiques sur le couloir de la piste d'athlétisme.

\subsection{Le suivi psychologique : l'investigation de la dimension affective de la problématique Psychologique}

Le type de prise en charge proposé à Emma est adapté au contexte de la préparation psychologique. Dans ce type d'intervention, le matériel clinique est fourni par la problématique sportive et sa signification préconsciente. La complexité du matériel permet d'autres types d'analyse. Il ne sera abordé que sous un certain angle permis par les limites du cadre instauré. Le but du clinicien est dès lors de fournir un cadre, un espace-temps : « Le travail du thérapeute vise à amener le patient d'un état où il n'est pas capable de jouer à un état où il est capable de le faire. » (Winnicott, 1977). Il s'agit donc pour le sportif de revisiter ses expériences à travers l'expérience partagée du jeu, d'oser mettre en désordre son fonctionnement psychique pour mieux le réagencer. Dans cet espace commun et partagé entre le sujet et le praticien, «l'action » est une mise en acte contrôlée. «La psychothérapie se situe en ce lieu où deux aires de jeu se chevauchent, celle du patient et celle du thérapeute. »

Dans cet espace potentiel à la croisée des réalités interne et externe, le sujet peut alors mettre en sens son expérience vécue sur le terrain sportif. Dans ce cadre contenant exempt de tout jugement, aucune interprétation n'était proposée pour ne pas empiéter sur le monde 
interne d'Emma. Ce qui est rejoué à un effet cathartique dont la signification n'est pas interprétée pour des raisons théoriques. Ainsi que Winnicott le spécifie, l'interprétation de contenu nous semble contre-indiquée dans ce type de consultation. Comme le signalait Dolto (1981) pour d'autres contextes d'intervention, le clinicien au sein du cadre de la préparation psychologique doit se préserver de faire de la «sous-psychanalyse » au risque de faire émerger des éléments qui ne peuvent pas être traités dans ce cadre et de bousculer l'équilibre psychologique du sujet sans pouvoir lui donner la possibilité de les reconstruire.

Ces rencontres et ces confrontations entre le psychologue et le sujet s'organisent autour des relations intersubjectives et de leur dimension affective réactivée au cours des échanges. Dans les phénomènes transitionnels qui s'établissent, Emma exprime ce qu'elle ressent. Dans ses demandes pressantes d'indications ou d'explication, je lui fais remarquer que dans sa construction le processus suit son cours. Qu'il n'y a pas de solution toute prête comme les consignes techniques en situation d'entraînement. Que la meilleure solution si elle existe est la sienne, celle qu'elle apportera lors du prochain entretien. Confronté à la frustration, Emma est sceptique et réagit de façon troublée face à cette nouvelle autonomie embarrassante. Déstabilisée par l'absence de prescription et de conseils, Emma est condamnée à se prendre en charge pour apporter ses propres réponses à ses propres questions étayées et orientées par le psychologue, à la différence de l'encadrement sportif qui les assiste dans leurs actes et décisions. Cette attitude est la première condition pour favoriser l'autonomisation et aider l'athlète à assumer ses choix. Dès lors, il semblait important de favoriser cette autonomie et ne pas proposer une interprétation forcée et plaquée, mais de jouer avec le temps et la maturation psychique. Emma me paraissait apte à pouvoir réaliser ce passage dans le cadre du dispositif psychologique.

\subsubsection{La dynamique des rencontres durant le suivi psychologique}

Après les entretiens préliminaires en salle où se détermine la problématique du sujet, les rencontres ont lieu en grande partie sur le terrain. Sans en avoir l'apanage, une des spécificités du psychologue du sport est que la dynamique des rencontres se déroule dans les différents lieux de pratique de l'athlète : salle, terrain (entraînement et compétition). Le psychologue du sport observe le sujet aux prises avec la réalité de la situation dans son environnement physique, social et institutionnel afin que se déposent en lui des indices lui permettant de mieux cerner la problématique individuelle. Chacune de ces analyses s'opère à plusieurs niveaux pour permettre une investigation plus fine des éléments récoltés et du matériel recueilli (observations, entretiens, tests...). Les observations5 permettent de recouper les informations afin de proposer un dispositif adapté et évolutif avec la dynamique psychique du sujet et de se rapprocher ainsi des conditions véritables de pratique pour mieux dépasser les problématiques en situation réelle de compétition.

L'observation montre qu'Emma est très avenante. Elle va au-devant des autres toujours le sourire aux lèvres avec un besoin marqué d'établir de nouvelles relations. Elle dit avoir besoin d'être acceptée et d'être aimée et qu'elle a peur de décevoir. Elle n'arrive pas à s'affirmer surtout pour exprimer ses compétences et ses qualités. Elle considère que c'est être « vantard » de se valoriser. Elle commence à évoquer ses qualités la séance suivante quand je lui demande de considérer celles qui lui ont permis d'atteindre ce niveau. Toutefois, nos entretiens sont centrés principalement sur la dimension sportive. Lorsque j'aborde des aspects plus personnels, elle replace son discours sur le terrain dans des considérations techniques. Elle ne semble pas encore prête à aborder des aspects plus intimes. Les rencontres suivantes, Emma fait part de son blocage sur les haies. Elle exprime ses difficultés : «Je me prends la tête. Y'a rien à faire », «Je me vois pas et pourtant je comprends ce qu'il faut faire, mais 
j'arrive pas à le faire ». L'état de doute s'instaure, elle le formule sous le terme de perte de ses repères en reprenant les mots de sa discipline sportive. En effet, si certaines épreuves s'améliorent, elle n'arrive pas à dépasser son problème de haie. Je lui fais part que cette répétition, ce blocage considéré comme absurde et à éluder, peuvent au contraire être perçus comme porteurs de sens.

3.3.2. Le passage d'un discours techniciste à un discours subjectif : l'investigation de la problématique psychologique

Dans une perspective psychodynamique, les premiers entretiens d'examen psychologique seront poursuivis par des entretiens d'approfondissement. Ces derniers se sont déroulés de fin avril à fin juin avant les grandes échéances compétitives, à raison d'une séance toutes les semaines. Le terme des consultations est défini par avance dans le contrat. Protégée par ce contenant temporel, Emma sécurisée va s'autoriser à investir ce suivi psychologique. Dans le cadre du dispositif, le sujet peut mettre en sens et ainsi dépasser ce point de butée. Dans une impasse, Emma ose dans un premier temps se risquer à une mise en mot de ses difficultés afin de mettre de l'ordre dans ce désordre dans l'espoir de passer ce cap. Emma est alors conviée à verbaliser son vécu et son ressenti. Progressivement, ce qui était considéré comme indigne d'intérêts est nommé et organisé dans un discours non plus techniciste mais subjectif. Elle commence dès lors à se réapproprier ce qui auparavant lui échappait. Au fur et à mesure des rencontres, un glissement s'opère des problématiques sportives vers des problématiques psychiques intimes émergentes.

Ce travail d'élaboration est fastidieux et s'effectue dans un cadre favorable où le sujet peut se risquer sur des chemins de traverse afin de pouvoir sortir des sentiers battus. Pas à pas, elle se décentre des considérations techniques pour enfin saisir l'intime sentiment et ses émotions jusqu'ici négligées. Au sein de l'institution sportive, la contrainte temporelle est importante. Le psychologue doit ajuster ses consultations en fonction du calendrier sportif très chargé avec des entraînements, des déplacements, des stages et des échéances compétitives. Pour ces raisons, la fréquence des entretiens est idéalement d'une fois par semaine, dans la pratique une fois toutes les deux semaines est un bon compromis.

\subsubsection{Les passages d'Emma}

Lorsqu'elle approfondit sa difficulté de passage de haie, elle articule sa problématique sportive technique à des difficultés affectives personnelles. Ces problématiques sportives et personnelles entrent en résonance et la renvoient à des conflits personnels déplacés et réactivés sur le terrain sportif dans la symbolique du passage de haie. Il ne s'agit pas d'écarter ou de mesurer ce qui gêne mais de le faire entendre, de faire en sorte que le sujet parle de ce blocage afin d'en faire surgir le sens à lui-même inconnu. Au fur et à mesure, qu'Emma ose les aborder, les problématiques psychologiques se révèlent dans la mouvance des changements vécus pendant cette période charnière de l'adolescence. Au cours de la dynamique des entretiens, Emma relie sa difficulté de passage à différentes difficultés singulières de transitions qui peuvent être organisées en six dimensions étroitement intriquées. Cette problématique de transition s'articule dans le cas d'Emma selon la symbolique du passage de l'adolescence à l'âge adulte, des changements de continent et de culture, de famille, d'entraîneur, de club et de statut. 


\subsubsection{Le passage de l'adolescence vers l'âge adulte.}

Cette vignette clinique met en évidence la problématique de la maturation qui s'avère être paradigmatique des adolescents sportifs de haut niveau. À une période de remaniements psychiques profonds et de bouleversements pour tous les adolescents, Emma en tant qu'adolescente sportive est de plus confrontée aux contraintes sportives qui potentialisent cette période de profonde mutation dans tous les domaines de la personnalité. L'adolescence dans son questionnement sur l'accès à l'autonomie représente un moment de crise identitaire qui doit conduire le sujet à abandonner des modèles extérieurs auxquels il s'est référé durant l'enfance pour accéder au sien. Paradoxe de l'adolescente qui cherche à rompre avec ses liens de dépendance tout en ayant besoin de ces étayages.

Cette période est une phase clé dans le processus d'autonomisation et dans la construction de repères identitaires. Les périodes de rupture sont nécessaires au changement et à la base même du développement de l'adolescente. Mais ces phases d'incertitude confrontent Emma à ces doutes, ses failles identitaires qui s'originent dans la prime enfance et qui sont réactivés dans cette période critique. L'adolescente devra oser le pas, prendre le risque de se désengager des images parentales. Pour Emma, les enjeux narcissiques se trouvent décuplés dans l'investissement compétitif. Le rapport du sujet à la compétition est essentiellement tributaire du rapport du sujet à la perte. La défaite comme la victoire la ramènent à la perte et dans sa dimension plus radicale à la castration. Ses expériences précédentes peuvent réactiver l'angoisse d'être confronté à nouveau à la perte et aux séparations subies durant l'enfance. Savoir se dégager du désir des autres, du regard d'autrui et accepter de ne pas plaire à tout le monde est le commencement sur le chemin de la performance. De sa réussite à se démarquer de l'emprise du désir des autres dépend son engagement comme sujet de son propre désir sur la voie de sa réalisation dans l'acte sportif.

Emma doit s'inscrire dans la perte de cette toute puissance, dans la dimension du manque à être et non de l'avoir pour atteindre la performance. Cette toute puissance magique du temps de l'illusion d'une saisie de la totalité doit être suivie pour Winnicott par le temps de la désillusion. Dans cette dialectique illusion-désillusion s'opère la reconnaissance d'une incomplétude dans la dimension du manque à être, même si le sujet n'a de trêve de retrouver cette toute puissance de perfection narcissique. C'est en effectuant ce passage qui implique un rapport au manque, à la perte et à la castration qu'Emma pourra affronter les compétitions d'une manière moins anxiogène. Elle doit donc se réapproprier son désir et accepter l'idée de perdre pour pouvoir gagner sa place. Cette difficulté du passage chez Emma témoigne des tentatives d'une remédiation des rapports intrapsychiques. L'ancien type d'appréhension et donc de gestion du réel n'est plus efficace face aux nouveautés, il ne permet plus l'adaptation aux changements successifs. Entre l'ancien et le nouveau, Emma est dans l'impasse : elle est dans l'indécision et le doute, étape nécessaire qui peut être constructive dans le cas d'une ébauche de nouveaux processus. Ce passage se situe entre le connu et l'inconnu, entre la réalité établie et le réel des changements (situations, statut, corps...). Dans cet entre-deux, le lien peut être établi dans le cadre du suivi psychologique.

\subsubsection{Transition de continent et de culture : d'un continent à un autre, d'une culture à une autre.}

Arrivée à l'âge de 13 ans en France, Emma est adoptée par une famille française tout en restant en relation avec sa famille d'origine en Afrique : "J'ai gardé un pied là-bas et j'ai un pied ici ». L'exil est un des déterminants historiques de la problématique actuelle d'Emma. Emma transporte avec elle dans son passage d'un continent à un autre, d'une culture 
à une autre, une part de son ailleurs inscrit dans sa problématique psychologique présente. Elle est confrontée à des référents culturels différents pas toujours évidents à concilier. L'expérience de l'exil pour Emma engendre de nombreuses pertes : perte de sa culture, de son statut, rupture des liens familiaux et sociaux. L'exil est assimilé à un véritable travail de deuil nécessitant un remaniement affectif et cognitif. Si le lien entre Emma et ses parents a été évoqué, il n'a pas pu être approfondi. Cette évocation provoquait la condition qu'Emma puisse commencer à se saisir du sens de sa filiation. Mesurer les incidences cliniques de «l'exil » sur la subjectivité et le mythe individuel qu'elle reproduit sans en avoir peut-être la moindre idée restait une tâche ardue dans les conditions limitées de ce suivi psychologique. Tobie Nathan dit qu'il n'y a de réalité psychique qu'ancrée dans une culture. Les risques cliniques pour le praticien, construits dans sa culture, sont multiples. Il peut plaquer ses représentations sociales et culturelles à son interlocuteur qui n'est pas dans la même mouvance culturelle ou alors évacuer le culturel dans une mise à distance des rapports de l'individu avec sa culture d'origine. Dans une certaine mesure, la démarche clinique voulait commencer à dégager le sens de son engagement et afin de comprendre les versions «culturelles » émises, c'est-à-dire le scénario porteur «d'explications » de l'histoire interculturelle. Pour Emma, ce changement de culture la confronte à l'inconnu et à la perte de repères. Emma a besoin de donner du sens à sa problématique, de l'intégrer à son histoire, de se la représenter tout en tenant compte de ses particularités culturelles. Écoutée, aidée, elle peut devenir elle-même l'artisan de sa propre expertise et commencer à investiguer sa problématique psychologique codée culturellement. L'attention clinique se porte sur la fonction de création de compromis et de liaison entre des univers hétérogènes que représente et qu'accomplit cette difficulté de passage. Pourtant, ce problème n'est pas infranchissable car pour Emma, l'amour des uns n'est pas porté au détriment des autres et l'entente se fait à son bénéfice. Ainsi, le passage de haie mettrait en échec des jeux de transfert entre l'origine et l'accueil, le passé et le présent. La problématique psychologique serait aussi une tentative de mise en tension entre des univers culturellement hétérogènes.

C'est la fonction du passage de haie de remettre en cause les espaces de transition qui peuvent se rejouer au sein de l'espace transitoire du cadre clinique. La posture du psychologue est alors de tenir compte de ses différences culturelles et de ne pas projeter son singulier comme universel produit d'un effet de la relation contre transférentielle dans sa dimension culturelle. Cette dernière contraint le psychologue à être attentif aux conséquences psychologiques de l'exil et à s'interroger d'autant plus sur sa pratique et ses représentations.

\subsubsection{Alternance de famille et de milieu de vie.}

Éloignement familial, séparation avec les amis et le copain ou la copine, si ces séparations peuvent être fécondes, elle est en l'occurrence brutale dans une situation qui nécessite un soutien. Ces ruptures ramènent Emma aux sentiments de séparation et de manque. Ces épreuves d'adoption et d'exil ont pu provoquer des expériences précoces pénibles génératrices d'angoisse. Ces angoisses se manifestent dans son cas par d'importantes fluctuations de l'estime de soi sur fond de doute quant à sa valeur sportive et à la conquête de la place qui lui est assignée. Angoisse d'autant plus prégnante qu'elle signifie pour Emma la perte de l'amour parentale si elle ne réalise pas les attentes des parents adoptifs. Ancien rugbyman, son père adoptif a favorisé sa pratique sportive tout comme sa mère. "C'est mes parents qui m'ont poussé à faire du sport. » Le père adoptif espère ainsi se réaliser à travers sa fille qui devrait accomplir son désir de haut niveau jamais atteint par lui-même. En retour, Emma souhaiterait combler ses parents adoptifs afin de ne pas perdre auprès des siens cette image héroïque. Image fantasmatique liée aux investissements libidinaux de son enfance et à sa toute puissance infantile. Elle devient l'objet de ce désir irréalisé. Elle est amenée à se constituer 
dans ce désir qui lui impose d'obtenir des résultats sous peine de perdre l'amour de ses parents. Si Emma parle moins de sa famille d'origine, elle n'a pourtant pas de difficulté à évoquer ses deux familles. Elle semble avoir bénéficié de cet amour de manière complémentaire et ne rejette aucune de ses familles au détriment de l'autre. Dans le cours des entretiens, ses propos n'évoquent aucune amertume explicite à l'égard de ses familles : «Ils se connaissent et s'entendent très bien ». L'amour parental semble être partagé même si elle aborde peu ses relations avec ses parents de «là-bas ». Elle semble toutefois en transition entre « l'avant et là-bas » et « l'ici et maintenant », le lien ne semble pas évident depuis ce nouveau changement avec son installation dans le centre d'entraînement. " Ici, j'ai pas vraiment d'amis, c'est plus des collègues. Mes amis et mes parents me manquent. » Face à tous ces remaniements, les mécanismes de défenses vont être mobilisés pour rejeter hors de la conscience des évocations ou des représentations porteuses d'angoisse. Dans le cas d'Emma, l'assomption de cette angoisse conséquente au manque perçu ou à venir conduit à la mise en place de mécanisme de défenses comme le déni. Dans un premier temps, elle annule tout effet de ses événements passés sur sa situation actuelle avant d'y revenir mais en minimisant leur portée. Dans l'urgence de l'adaptation imposée, il lui est certainement difficile de s'y confronter et de remanier des défenses sans l'assurance de pouvoir les reconstruire. Elle évoquera cette transition de milieu de vie selon trois périodes. La première période de changement de lieu de vie et d'éloignement du cadre familial. Elle se retrouve dans une période passagère de solitude l'incitant à réinvestir de nouvelles relations. "En plus, on n'a pas de loisirs et comme je connais personne c'est dur surtout les week-ends. Les autres rentrent chez eux, moi c'est trop loin. » L'entrée au pôle d'entraînement correspond au premier éloignement de la structure familiale et de ses amis qu'elle ne rejoint pas souvent. Dès lors la confrontation entre la représentation idéalisée du sport-études et la réalité institutionnelle nécessite un remaniement de ses représentations. Suit une seconde période où l'habitude et l'usure engendrent la lassitude de la répétition des contraintes scolaires, sportives après une période de découverte des possibles. Enfin, la prise de décision et l'établissement de nouveaux repères face aux différentes opportunités chargées d'inconnues. "L'internat me change. Moi, j'ai pas encore trop de problèmes, mais l'année prochaine, je demande à prendre un appartement car j'en peux plus. »

\subsubsection{De l'entraîneur au substitut parental.}

Dans ce passage de l'entraîneur au substitut parental, Emma effectue un remaniement de ses relations avec son entourage, ses parents mais aussi avec la personne la plus proche dans l'immédiat, son entraîneur. La relation privilégiée entraîneur-entraîné influence l'investissement sportif de la part de l'athlète. L'émergence d'une dyade entraîneur-entraîné se fonde sur le primat d'une relation affective et non sur les seuls aspects fonctionnels, cognitifs ou instrumentaux (Lévêque, 2005). Une dyade désigne une nécessité réciproque de l'un à l'autre pour exister où chaque individu est différent de ce qu'il est séparément. En ce sens, la dynamique de la relation entraîneur-entraîné est liée non pas seulement à des facteurs opérationnels mais aussi et peut-être surtout à l'imbrication des besoins pulsionnels et identificatoires réciproques. L'entraîneur a un poids important dans les décisions et l'évolution de sa carrière sportive. «Mon premier entraîneur d'athlétisme m'a inscrite au déca ; mais après, avec mon entraîneur ça n'allait plus, ça devenait étouffant, il me criait dessus quand j'avais pas de bons résultats. Je l'ai dit à mes parents qui ont téléphoné à mon nouvel entraîneur qu'ils avaient vu à une compétition. » Emma éprouvait des réticences à se confier à son entraîneur qui faisait figure de substitut parental et d'autorité. Elle cherche ses repères entre les entraîneurs de la nouvelle section et celui de son ancien club. Les effets de la 
puberté peuvent avoir des conséquences sur les identifications successives à ses substituts parentaux et la relation entraîneur-entraîné. Emma met son entraîneur à la place de son idéal du moi. Son entraîneur incarne un idéal qu'elle veut atteindre, Idéal graduellement établi au confluent de son narcissisme et de ses identifications précédentes intériorisées comme référentielles. En prenant la place de l'Idéal du moi laissé vacant par les parents, l'entraîneur devient de la sorte un substitut parental. Qualifié de « binôme s'autoengendrant, interdépendant, inséparable et pour lequel tout mouvement de l'un des partenaires rejaillit d'un système à économie fermé [...] cet équilibre dans l'interdépendance peut être remis en question par le processus de l'adolescence qui dérange le sportif dans son identité (Carrier, 1992). » L'entraîneur vient en prolongement par rapport à la structure familiale (Labridy, 1989). Il vient combler le manque ou la perte de ce qui a manqué dans les relations familiales. D'autant plus que pour Emma, la figure du père absente dans un premier temps du fait d'un abandon précoce est l'objet d'une idéalisation favorisant l'investissement de la personne de l'entraîneur. L'entraîneur sans en avoir conscience, se propose de prendre la place d'un autre manquant. Ce rapport à l'être absent permet à l'entraîneur de s'inscrire et de trouver sa place dans une chaîne relationnelle institutionnelle et familiale. Cette hypothèse d'une transmission générationnelle va s'opérer à partir de la faille et du manque à être propre à son histoire personnelle d'exil et d'adoption. Cette proposition qui lui est faite de trouver enfin sa place séduit Emma. La jeune sportive est confrontée à une relation affective forte avec son entraîneur qui partage sa vie quotidienne, ses déplacements, ses espoirs et ses idéaux. " $\mathrm{Ce}$ n'est pas tant ce qu'il dit que la place à partir de laquelle il parle qui donne du poids et de l'effet à sa parole (Ragni, 1990). » Cette notion de dépendance dans cette relation est mise en exergue par Lévêque, (1987) : «La relation entraîneur-entraîné est restée très verticale et fonctionnelle, l'attitude pédagogique se fonde encore trop souvent sur une stricte hiérarchisation des compétences et le maintien d'un lien fonctionnel de dépendance ». Le cadre clinique peut favoriser le dégagement d'une relation fusionnelle par l'instauration d'une distance et permettre ainsi à la confusion entre soi et l'autre de se discerner dans un processus d'accès à l'autonomie.

\subsubsection{Transition de clubs et d'environnement institutionnel : du club local au pôle France.}

Emma se retrouve dans un autre monde, sans que ses anciens mondes disparaissent. L'environnement social et institutionnel impose une nouvelle adaptation à la jeune sportive. Pour elle, la structure, la hiérarchie et les règles institutionnelles en internat infligent un mode de vie radicalement différent par rapport à la structure familiale. Pour réussir dans le milieu sportif, il lui faut s'adapter à ces nouvelles conditions de vie. L'environnement institutionnel fournit à l'adolescente une nouvelle identité fondée sur un idéal social du champion. Cette identité sociale valorisante et valorisée dans laquelle elle peut se projeter peut devenir aliénante et perturber l'évolution et le développement de l'adolescent sportif de haut niveau. " L'adhésion de l'adolescent à ces propositions lui permet de ne pas encore affronter son propre « passage » à l'âge adulte [...]. Il risque cependant de rester fasciné par ses titres de gloire ou même séduit, de confondre son identité d'adulte, virilité et identité sexuelle... Alors, entravée la poursuite de ses investissements et de son développement psychique en tant que sujet autonome (Carrier, 1992). » Le risque pour l'adolescente sportive est de ne pas échapper aux arcanes des représentations sportives et des identifications forcées, isolée qu'elle est dans ce monde avec ses propres valeurs. Le monde sportif est un microcosme d'où la jeune athlète a du mal à s'extraire.

Ce monde dans le monde à ses propres règles auxquelles le jeune se soumet souvent s'en rendre compte. Prendre de la distance en prenant conscience dans un premier temps de ses enjeux cruciaux pour son développement est fondamental dans la structuration de l'adulte. 
Mais le monde sportif présente aussi l'avantage et l'inconvénient de générer un système de fonctionnement où les athlètes ont peu l'occasion de se prendre en charge personnellement. L'entourage (sponsors, dirigeants, entraîneurs, équipe) «gère leur quotidien » qui est planifié et déterminé du fait des contraintes et de l'activité propre au monde sportif. «La focalisation de toutes les énergies sur l'objectif sportif est particulièrement manifeste dans l'organisation du mode de vie à l'intérieur du microcosme du sport. Surveillants, animateurs, professeurs, entraîneurs, médecins sont là pour satisfaire, prendre en charge, stimuler les athlètes. Ces derniers sont ainsi assistés par un environnement qui anticipe les réponses à toutes les questions. Ayant tout sur place, ils n'ont ni temps, ni loisir pour formuler des tensions désirantes différentes du sport (Carrier, 1989). " Au risque de s'aliéner aux normes institutionnelles, Emma doit s'affranchir des idéaux institutionnels en fondant ses propres valeurs. Au cours des entretiens, elle se cherche, comprend ce qu'elle ne veut pas, mais ne sait pas encore ce qu'elle veut, ce qu'elle est : «je sais pas très bien encore ce que je veux ». La maturation psychologique est un processus très progressif pendant l'adolescence période de tous les changements. Ces bouleversements engendrent parfois des crises qui rendent possible les modifications du comportement, des systèmes de défense, des schémas de représentations et d'actions. Le dispositif psychologique peut fournir à Emma les conditions d'une crise maturante. Il offre un espace transitoire pour se construire, s'autoriser à se poser les questions et éventuellement les élaborer en testant la potentialité des différents choix identificatoires. Il permet ainsi des réinvestissements sur de nouveaux objets fondés sur ses propres désirs afin que son projet puisse apparaître.

\subsubsection{6. Évolution de niveau et de statut : du statut d'espoir au statut de haut niveau.}

À la problématique de passage d'une identité d'adolescent à celle d'adulte se surajoute celle d'une identité de sportif à une identité de sportif de haut niveau. Les adolescents sportifs sont soumis à la fois aux processus de développement physiologique, psychologique et social ainsi qu'à l'exigence du haut niveau qui nécessite de concilier les études avec les entraînements et les compétitions sportives au détriment souvent des loisirs et parfois de son épanouissement personnel. Les conditions de vie du sport de haut niveau imposent ses propres contraintes : emploi du temps surchargé, éloignement familial, déplacements fréquents et parfois longs, fatigue, lassitude, exigence de la performance, dépassement de soi et maîtrise du corps. Ce régime contraignant peut déstabiliser l'adolescente déjà engagée dans une période d'instabilité notoire dans son évolution. Les conséquences d'une perte de repères sont l'expression de son impossibilité à combiner l'exigence de la réalité, c'est-à-dire pour Emma à trouver sa place en fonction de son niveau de pratique sportive et de ses compétences, à assumer ce nouveau statut prometteur, à sa volonté d'avoir un niveau égal voire supérieur aux athlètes qui s'entraînent depuis des années. «Je pensais pas faire partie du pôle France. J'ai jamais su pourquoi j'ai été accepté dans l'équipe. Je dois démontrer pourquoi je suis là. Ici, ils ont un meilleur niveau que le mien, je suis arrivé dans un autre monde. » Avec son organisation spécifique à un milieu productif soumis à la performance, l'institution sportive engendre une somme de contraintes et de pressions multiples exercées sur l'adolescent sportif de haut niveau. Dans cet environnement spécifique, l'accumulation de ces changements déstabilise Emma dans une période critique. La perception et l'évaluation de ces variations par le sujet jouent un rôle considérable dans l'évolution de ce processus dynamique. Son histoire singulière reliée au rôle et aux attitudes de ses parents et de ses proches, au cursus scolaire ainsi qu'aux événements sportifs contribue à la difficulté de passage de cette période critique. De fait, l'inconnu du changement est une effraction du réel qui réactive des conflits antérieurs. Mais en même temps, il met en exergue la tentative du sujet d'ébaucher une remédiation des rapports intrapsychiques et le monde extérieur. Emma doit constituer son sujet sportif dans 
l'intersubjectivité (ses pairs sportifs, ses modèles, son entraîneur), mais aussi sur ses propres désirs. Un des rôles du psychologue dans cette approche clinique est d'accompagner ces sportifs pour que les choix et les pertes qui marquent et jalonnent leur vie dans ces périodes de transition ne les perturbent pas autant dans leur pratique et dans leur vie. En l'occurrence, il s'agissait d'aider Emma à se démarquer de cette imposition d'un idéal social de performance, à se dégager des représentations imaginaires collectives et lui permettre de s'approprier le sens de ses actions par la compréhension de ce qu'elle vit. De lui permettre d'articuler la logique institutionnelle de l'excellence avec sa logique de sujet sportif, de dépasser une réalité technique et tactique pour l'intégrer à l'imaginaire et à la symbolique de son vécu.

\subsubsection{Le passage de haies : une problématique psychologique à dépasser}

Dans le cadre du suivi psychologique, l'objectif était d'aider Emma à trouver ses repères et ainsi restaurer une confiance en soi passagèrement altérée par les épreuves qu'elle traversait. En lien avec le paradigme de passagèreté (Martineau, 1993), l'évolution d'Emma pourrait conjuguer son processus de passage en trois temps qui désigneraient les trois étapes qui se jouent au sein du cadre d'intervention psychologique. Dans un premier temps, le sujet marque le pas. Il est confronté à son obstacle dans l'impossibilité de le dépasser. Dans le deuxième temps de la passe, il lui faut alors faire un pas de côté pour se démarquer des processus répétitifs qui ancrent le sujet dans l'échec. Le sujet dénoue alors le paradoxe et sort de l'indécision, de l'impasse. Il est alors capable de s'engager dans le passage et de trouver une ouverture dans le troisième et dernier temps. Il peut enfin dépasser le seul résultat comptable pour se dépasser, se transcender dans le passage qui le consacre dans l'acte créateur et enfin performer. Dans le champ du sport de compétition, cette exigence de la prise en compte de la performance sportive au sein de cette logique institutionnelle de résultats avec son corollaire, l'urgence et dans le meilleur des cas le court terme, confond performance et résultat. La performance comme nous l'avons appréhendée ne se réduit pas au résultat comptable, quantifiable et mesurable. Cette logique rend difficile la construction d'un projet de prise en charge psychologique s'inscrivant dans le temps et tenant compte de la performance c'est-à-dire de la réalisation du sujet sportif. Nous supposons que l'hypothèse d'un déplacement de ses conflits psychiques sur le couloir de la piste d'athlétisme engendré pour Emma par son désir de performance serait partiellement confirmée. Hypothèse que nous avons appréhendée au travers des multiples passages qu'Emma a traversés sur le chemin de sa réalisation et de sa performance. Ses passages et ses difficultés ont été abordés comme une transition entre l'inadaptation des anciens rapports et la tentative d'établir de nouveaux rapports pouvant déboucher sur une résolution de la crise. Ainsi, les problématiques de passage s'articulent dans une constante évolution au cours du développement d'Emma. Ce développement est favorisé par le cadre du dispositif de préparation psychologique. Dans ce cadre, le rôle du psychologue dans le champ du sport est d'appréhender les phénomènes humains selon une perspective qui donne à la complexité une place privilégiée. C'est-à-dire saisir le sujet dans sa dynamique singulière au sein de la globalité de sa situation. Cette interaction entre le sujet et la situation réactive des conflits psychiques antérieurs sous l'effraction réelle, symbolique et imaginaire de l'environnement sportif (Lecocq, 1997).

\section{Conclusion}

Cette vignette clinique n'a pas la prétention de servir de généralisation à d'autre cas. Elle veut rendre compte d'une pratique dans le contexte particulier de la compétition sportive de haut niveau. Ce compte rendu de pratique souhaite faire percevoir l'évolution du dispositif psychologique qui s'élabore dans les rencontres partagées avec l'athlète mais aussi avec l'entraîneur en interaction avec un environnement institutionnel et physique influent. Le 
dispositif s'inscrit à la fois au sein d'une procédure planifiée, mais également flexible pour s'adapter à la demande évolutive et à la dynamique psychologique du sujet. Les pratiques se créent sur la base d'une trame technique répertoriée mais elles doivent rester créatives dans la confrontation et la collaboration des multiples acteurs de la préparation psychologique. Cette tentative de formalisation d'un dispositif de préparation psychologique explicité selon des phases pour rendre claire et plus accessible l'exposé ne se veut pas rigide et figée dans l'application contextualisée sur le terrain. Pour Emma, le passage de haie devient la métaphore de ses conflits psychiques. Le déplacement de ses conflits internes qui se manifeste sur le terrain sportif. La contre performance qui se métaphorise dans le passage de haie signale son ambivalence face à son histoire personnelle que le remaniement dans ses dimensions personnelles, relationnelles et environnementales peut aider à dépasser. Grâce au temps et aux soutiens qu'elle a sollicités, Emma a su retrouver ses appuis et ses repères. Reste la difficile question de l'évaluation du dispositif et de son rôle dans l'évolution d'Emma. Cette mise en sens de la problématique sportive dans le cadre du suivi psychologique allié à l'approfondissement de la technique de préparation mentale a pu contribuer à ce passage. De l'avis de l'intéressé, cette pratique psychologique s'est révélée être une méthode « enrichissante ». Elle se sentait active et à l'origine de toute la démarche. Elle a pu «mettre à plat des questions qui me prenait la tête. » et se sentir soutenue et écoutée. Si on ne connaît pas la part de bienfait du dispositif, néanmoins la prise en charge précoce a pu contribuer au passage de ce blocage et éviter que celui-ci perdure et s'aggrave. Dans ce cas, il s'agit bien d'une préparation qui cherche à anticiper les problèmes plus graves et ainsi éviter la réparation. La préparation psychologique s'inscrit dans une visée préventive et prophylactique qui s'efforce de prévenir les défaillances et d'aider à dépasser les difficultés d'adaptation psychologique inhérentes à la haute performance.

Cet exemple clinique peut apporter à travers cette lecture et cette analyse, des éléments de compréhension qui ont pu contribuer à une meilleure représentation de ces interventions afin de mieux comprendre l'importance des enjeux et des services qui devrait être accessible à tous ceux qui le souhaitent. L'enjeu de cette réflexion souhaite apporter un autre éclairage à ce type de dispositif psychologique par la mise en exergue du vécu de ces sujets et de l'importance du suivi psychologique notamment dans le cas des adolescents sportifs.

\section{Références}

Carrier C. (1989). Le sportif face aux phénomènes d'attente collective. Actes du IIe colloque de L'Insep, GREPAS, 49-53.

Carrier, C., 1992. L'adolescent champion. PUF, Paris.

Dolto, F., 1981. Au jeu du désir, essais cliniques. Le Seuil, Paris.

Fernandez, L., Catteeuw, M., 2001. La recherche en psychologie clinique. Actualités et nouvelles perspectives en psychologie clinique. Nathan Université, Paris.

Greenspan, M.J., Feltz, D.L., 1989. Psychological interventions with athletes in competitive situations: A review. Sport Psychologist. 3 (3), 219-236.

Labridy, F., 1989. Le couple entraîneur-entraîné : activité passivité. In: Actes colloque : sport et psychanalyse, l'investissement sportif de haut niveau dans la compétition et l'entraînement. Insep publications, pp. 81-85. 
Lecocq, G., 1997. Le psychologue du sport : un médiateur de sens entre la préparation mentale, le sportif et son environnement. In: L'entraînement mental et sport de haute performance. INSEP publications, pp. 27-40.

Lévêque, M., 1987. In: L'adaptation psychologique à la pratique sportive intensive. Sport et santé mentale, Bruxelles, pp. 21-31.

Lévêque M. (1993). Sport et psychologie : l'apport du psychologue aux acteurs. Actes des entretiens de l'Insep.

Lévêque, M., 2005. Psychologie du métier d'entraîneur ou l'art d'entraîner les sportifs. Vuibert, Paris.

Martineau, J.P., 1993. L'effet de passe. In Quel corps ?. Sciences humaines cliniques et pratiques corporelles 2, 237- 244.

Nicolas, M., 2004. L'intégration d'une technique de préparation mentale dans un dispositif de préparation psychologique. Revue de psychologie du sport : second souffle 5, 67-80.

Nicolas, M., (2002). La professionnalisation des intervenants en psychologie dans le sport de haut niveau. Colloque international de management du sport. Centre national des sports du Maroc. Rabat-Salé, 8-9 mars 2002.

Nideffer, R.M., 1981. The ethics and practice of applied sport psychology. Movement Publications, New York.

Orlick, T., 1986. Psyching for sport: Mental training for athletes. Champaign (III). Leisure Press.

Pedinielli, J.L., 1994. Introduction à la psychologie clinique. Nathan, Paris.

Ragni P. (1990). L'extrême de la relation entraîneur - entraîné. Actes du IIe colloque sport et psychanalyse, octobre 1990, Insep publications, 59-65.

Resweber, J.P., 1995. La recherche-action. PUF, Paris.

Unestahl, L.E., 1983. The mental aspects of gymnastics. Veje Forlag, Ôrebro.

Vealey, R.S., 1994. Current status and prominent issues in sport psychology interventions. Medicine and science in sport and exercise (pp. 495-502).

Winnicott, D.W., 1977. Jeu et réalité. Gallimard, Paris. 